\title{
Barber as infectious agent
}

To the Editor: There are at least half a dozen barbers on the main streets or 'high roads' of suburbs such as Lenasia Ext. 1, Fordsburg and Mayfair in Johannesburg, South Africa. These are run by people of Pakistani, Indian and Bangladeshi origin. We have seen a proliferation of these businesses over the last few years; they provide men's haircuts for a cheap price of between ZAR25 and ZAR30.

As dermatologists and general practitioners, we have been seeing a higher than usual number of new patients with scalp and facial warts, fungal infections and eczematous rashes recently. The common history is frequent visits to the above type of barber, leading to our clinical and expertise-based diagnosis and conclusion.

A personal visit to a few of these little 'barber shops' led us to the opinion that the dermatological infections listed above are a direct result of transfer of infectious agents between clients. These hardworking barbers do use new 'sterile Minora' blades for shaving, which provide a false sense of security. They usually use methylated spirits to sterilise some of their tools of trade. This is not a hygienic method to sterilise equipment, as it is not sufficient to kill fungi, bacteria or viruses. On average, 20 - 40 people are seen in a day, and perhaps more over the weekend. This includes routine haircuts to fancy hairdos, shaving, and head and neck massages using shared oils (i.e. shared by many people).

The barber also does not wash his hands regularly. Skin infections with human papillomavirus and dermatophytes, and allergic contact, are probable clinical sequelae to exposure to these unclean practices.

The increasing incidence and difficulty in treating these dermatological conditions warrants further investigations into this possible public health problem so that appropriate action and changes in the above practices, such as better education of the barbers, can be taken and implemented.

D Modi , Z Modi

Division of Dermatology, Department of Internal Medicine, Faculty of Health Sciences, University of the Witwatersrand, Johannesburg, South Africa howzat@iafrica.com

\section{S Naidoo}

School of Public Health, Faculty of Health Sciences, University of the Witwatersrand, Johannesburg, South Africa 